\title{
Razón entre grasa visceral y subcutánea como predictor de alteraciones cardiometabólicas
}

\section{Ratio of visceral to subcutaneous fat as a predictor of cardiometabolic risk}

\section{ABSTRACT}

Visceral fat is an important predictor of cardiometabolic risk, but evidence suggests that the ratio between visceral and subcutaneous fat $(\geq 0.4)$ may be a more appropriate indicator to assess individual predisposition to accumulate visceral fat (VF) and predict cardiometabolic alterations. Objective: To evaluate the predisposition to accumulate $V F$ and its association with cardiometabolic alterations. Methods: A cross-sectional study was conducted with 147 patients seen at a hospital in Northeast Brazil. VF and subcutaneous fat (SF) were evaluated by computed tomography and anthropometric and cardiometabolic risk factors were investigated. Results: The mean age was $52.7 \pm 13.2$ years. Mean VF/SF ratio was high: $0.79 \pm 0.29$ for men and $0.54 \pm 0.22$ for women and was correlated with the glucose profile and coronary calcium score (CCA) $(p<0.05)$. VF was directly correlation with non-HDL cholesterol, triglycerides (TG), TG/HDL ratio, glycemia, glycated hemoglobin and CCA $(p<0.05)$ and inversely with HDL-c $(p=0.001)$. Conclusions: A high predisposition to $V F$ accumulation was observed in the population. VF/ $S F$ ratio correlated with cardiometabolic alterations, but the concentration of isolated VF correlated with a higher number of parameters. In this investigation, VF alone was a more powerful predictor of cardiometabolic alterations than the ratio of $V F / S F$.

Key words: Visceral fat, Subcutaneous fat, Abdominal obesity, Calcium score.

\section{RESUMEN}

La grasa visceral es una importante medida predictora de riesgo cardiometabólico, sin embargo, se ha sugerido que la razón entre los componentes visceral y subcutáneo (TAV/TAS $\geq 0,4)$ puede ser un indicador más apropiado para evaluar la predisposición para acumular grasa visceral (GV) y las alteraciones cardio-metabólicas. Objetivo: Evaluar la predisposición para acumular GV y su asociación con alteraciones cardio-metabólicas. Métodos: Estudio transversal con 147 individuos atendidos en un ambulatorio de un hospital en el Nordeste brasileño. Se evaluaron el TAV y el TAS por tomografía computarizada y se investigaron
Elíndice dos Prazeres Almeida ${ }^{1}$, Cláudia P. Sabino Pinho ${ }^{1,2}$, Ana Paula Dornelas Leão ${ }^{1}$, Isa Galvão Rodrigues ${ }^{1}$, Alcides da Silva Diniz², Ilma Kruze Grande de Arruda².

1. Pronto Socorro Cardiológico Universitário de Pernambuco,
Recife-PE, Brasil.
2. Universidade Federal de Pernambuco, Recife-PE, Brasil.

Dirigir correspondencia a: Claudia Porto Sabino Pinho. Rua dos Palmares, s/n, Santo Amaro, Recife-PE, CEP: $50.100-060$, Brasil. Fone: +558199635-6615. Email: claudiasabinopinho@hotmail.com

Este trabajo fue recibido el 26 de mayo de 2017. Aceptado con modificaciones: 20 de septiembre de 2017. Aceptado para ser publicado: 05 de noviembre de 2017.

variables demgráficas, clínicas, antropométricas y factores de riesgo cardiometabólico. Resultados: El promedio de

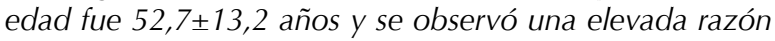

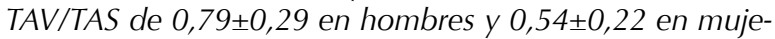
res. Se correlacionó la razón TAV/TAS con perfil glucídico y puntuacion de calcio coronariano $(P C C)(p<0,05)$, mientras que el TAV aislado presentó correlación directa con colesterol no-HDL, triglicéridos (TG), razón TG/HDL, glicemia, hemoglobina glicosilada y PCC $(p<0,05)$ e in- 
versa con HDL-c $(p=0,001)$. Conclusiones: Se observó una elevada predisposición para la acumulación de TAV. La razón TAV/TAS presentó correlación con alteraciones cardiometabólicas, pero la concentración de TAV aislada se correlacionó con un número superior de parámetros, siendo en esta investigación un predictor más potente que la razón TAV/TAS para indicar esas alteraciones.

Palabras clave: Grasa visceral, Grasa subcutánea, Obesidad abdominal, Puntuación de calcio coronariano.

\section{INTRODUCCIÓN}

La obesidad se puede definir como la acumulación excesiva de grasa corporal frecuentemente asociada a la salud deteriorada ${ }^{1}$. Actualmente se considera un problema de salud pública que sigue creciendo en todo el mundo, presentando proporciones epidémicas y siendo capaz incluso de disminuir la expectativa de vida de los individuos comprometidos ${ }^{2}$.

Los efectos devastadores causados por la acumulación de grasa corporal son varios, pero es importante destacar que no todos los individuos obesos y no todos los tipos de adiposidad confieren riesgo cardiovascular y metabólico equivalente ${ }^{3}$. Dependiendo de los lugares en los que hay deposición de grasa, se observan diferentes respuestas biológicas ${ }^{4}$.

El tejido adiposo abdominal incluye los depósitos de grasa subcutánea y visceral, los cuales presentan diferencias morfológicas y funcionales que confieren riesgos diferenciados sobre las alteraciones metabólicas y hemodinámicas 5 . La principal y más importante diferencia existente entre esos dos compartimentos de grasa es el drenaje venoso. Mientras el tejido adiposo subcutáneo (TAS) tiene su drenaje realizado por la circulación sistémica, el tejido adiposo visceral (TAV) es irrigado por la circulación portal ${ }^{6}$. Además, el TAV secreta mayores concentraciones de citoquinas inflamatorias, comparado al TAS y al tejido adiposo glúteo-femoral, lo que lo vuelve el componente que produce mayor efecto deletéreo sobre parámetros cardio-metabólicos ${ }^{5,6}$.

La cuantificación de la grasa visceral tiene gran importancia como medida predictora de riesgo cardiometabólico elevado. Además, la propensión a acumular preferentemente grasa visceral en condiciones de exceso de ingestión de energía es altamente variable de un individuo a otro. Sin embargo, su cuantificación absoluta puede no reflejar el riesgo de que el individuo sea visceralmente obeso, y se ha sugerido que la razón entre los componentes visceral y subcutáneo (TAV/TAS) puede ser un indicador más apropiado para reflejar la acumulación de grasa visceral y para evaluar la predisposición del individuo a acumular preferentemente grasa visceral 7 .

Así, se sugirió a la razón TAV/TAS como una métrica de grasa abdominal relativa y valores $\geq 0,4$ serían indicativos de una mayor predisposición a la acumulación de TAV ${ }^{7}$. Pocos estudios han evaluado la predisposición para acumular grasa visceral a partir de esta razón y su relación con alteraciones cardiometabólicas. Por tanto, nuestro objetivo fue evaluar la predisposición para la acumulación de grasa visceral a partir de la razón TAV/TAS y su asociación con alteraciones cardiometabólicas.

\section{MÉTODOS}

Estudio transversal, desarrollado en ambulatorio general de Nutrición de un hospital cardiológico, localizado en el Nordeste brasileño, con muestra captada entre 2013 y 2015, involucrando a individuos de ambos sexos, con edad $\geq 20$ años.

La muestra se selecionó en base a adhesión voluntaria, siendo excluidos los individuos con hepato y/o esplenomegalia, ascitis, cirugía abdominal reciente y mujeres embarazadas o que tuvieron hijos hasta 6 meses antes del rastreo de la investigación, ya que estas características podrían influir en la medida de grasa intraabdominal y/o en las medidas antropométricas. También se excluyó a individuos portadores de limitaciones físicas que imposibilitasen la medición de medidas antropométricas y a individuos con claustrofobia.

Teniendo en cuenta un error $\alpha$ del $5 \%$, un error $\beta$ del $20 \%$, una correlación promedia estimada entre la razón TAV/ TAS y el perfil glicolipídico de $0,5(p)^{8}$ y una variabilidad de $0,1\left(d^{2}\right)$, se obtuvo un tamaño de muestreo mínimo de 113 individuos. Para corregir eventuales pérdidas, se incrementó en un $30 \%$ ese número, totalizando un n de muestreo de 147.

EI TAV se evaluó por tomografía computarizada (TC) de abdomen sin contraste -método considerado de referencia para la cuantificación separada de los compartimentos de tejido intraabdominal- por un único médico radiologista observador, entrenado en el protocolo del estudio. El área del TAV se obtuvo por el equipo de tomografía Philips Brilliance CT-10 slice (VMI Industria e Comércio Ltda, Lagoa Santa, MG, Brasil) y el examen se realizó en ayuno completo de cuatro horas con el paciente en decúbito dorsal. Se obtuvo el corte tomográfico con parámetros radiográficos de $140 \mathrm{kV}$ y $45 \mathrm{~mA}$, en el nivel de L4, con espesor de $10 \mathrm{~mm}$. El área total de grasa abdominal total y el área de grasa visceral se delinearon manualmente con cursor libre contornando cada región. Toda la superficie de la piel se excluyó del área de marcado. El área del TAV se determinó tomando como límites los bordes internos de los músculos recto abdominal, oblicuo interno y cuadrado lumbar, excluyendo el cuerpo vertebral e incluyendo la grasa retroperitoneal, mesentérica y omental. El área de grasa subcutánea fue entonces calculada restando el TAV del área total de grasa. Todas las áreas de grasa se describieron en $\mathrm{cm}^{2}$. Para la identificación del tejido adiposo, se utilizaron los valores de densidad de -50 y -250 unidades Hounsfield 9 . Una razón TAV/TAS $\geq 0,4$ se estableció como punto de corte para indicar la predisposición de la acumulación de grasa visceral ${ }^{7}$.

Los análisis bioquímicos se realizaron en el Laboratorio de Análisis Clínicos del hospital, siendo considerado un ayuno de 12 horas y protocolo de preparación, según preconiza la 
V Directiva Brasileña de Dislipidemias, 2013. Se evaluaron los parámetros: glicemia de ayuno y hemoglobina glicosilada (HbA1C), el perfil lipídico (Triglicéridos (TG), colesterol total (CT) y fracciones, colesterol no HDL, ratio TG/HDL), teniendo como referencia de normalidad los valores propuestos en la $\vee$ Directiva Brasileña sobre Dislipidemia y Prevención de la Aterosclerosis, 2013.

La glicemia de ayuno y el perfil lipídico se analizaron por el método enzimático, y la hemoglobina glicosilada (HbA1c) por turbidimetría. Los análisis clínicos se realizaron utilizando un analizador Cobas Íntegra $400^{\circledR}$ (Roche Diagnostics). Se utilizó la fracción del colesterol no HDL como estimación del número total de partículas aterogénicas en el plasma $(\mathrm{VLDL}+\mathrm{IDL}+\mathrm{LDL})$. El colesterol no HDL se calculó por la resta: Colesterol no HDL= CT - HDL-C. La razón TG/ HDL-c se utilizó como índice de aterogenicidad por reflejar el tamaño de las partículas de LDL-c.

Como parámetros antropométricos se evaluó: circunferencia abdominal (CA) e índice de masa corpórea (IMC). Se recolectaron todas las medidas en duplicados por un solo observador y repetidas cuando el error de medición entre ellas era mayor que $0,1 \mathrm{~cm}$ ó $0,1 \mathrm{~kg}$. La medida final considerada fue el promedio entre los dos valores más cercanos.

La CA se obtuvo con una cinta métrica inelástica, con precisión de $0,1 \mathrm{~cm}$, directamente sobre la piel, en el punto medio entre la última costilla y la cresta ilíaca ${ }^{10}$. Los marcos óseos de la última costilla y de la cresta ilíaca fueron localizados y palpados por el examinador en el nivel de la línea axilar media. La cinta de medición se colocó en un plano horizontal alrededor del abdomen en los lugares descritos anteriormente y se prestó especial atención para garantizar que la cinta estuviera paralela al suelo. La medición se realizó al final de la expiración normal como cinta inelástica adyacente a la piel, pero sin comprimirla, con el participante en pie y bien erecto.

La calcificación coronaria se evaluó a partir del método de puntuación de calcio coronario (PCC) obtenido por TC con múltiples detectores. Por medio de ese método, se mapeó el corazón por un período de 20 a 30 segundos, sincronizado al electrocardiograma, con una distancia de $3 \mathrm{~mm}$ entre cada corte. Un software detectó lesiones de calcificación con una densidad de al menos 130 unidades Hounsfield (HU) y con un área mínima de $0,5 \mathrm{~mm}^{2}$. A partir de la fase diastólica del ciclo cardíaco, las imágenes fueron reconstruidas y el cálculo total de la calcificación fue determinado mediante fórmula cuyos componentes son las medidas de volumen total y del área total de la lesión calcificada. Las arterias mapeadas fueron: aorta coronaria derecha, tronco de la coronaria izquierda, arteria descendente anterior y sus ramas y arteria circunfleja y sus ramas. La puntuación total de la calcificación coronaria medida en HU correspondió a la suma de la puntuación de todas las arterias. La presencia de la calcificación coronaria se determinó cuando el resultado de PCC > 0 , siendo considerado ausente cuando el valor de PCC fue igual a cero' ${ }^{11}$.

Se analizaron los datos con la ayuda del programa SPSS versión 13.0 (SPSS Inc., Chicago, IL, USA). Se realizó un análisis exploratorio de los datos y las variables continuas fueron probadas en cuanto a la normalidad de la distribución, por el test de Kolmogorov Smirnov. Los datos de las variables de distribución normal se expresaron en la forma de media y desviación estándar (DE), aplicando las pruebas paramétricas para comparación (T de Student para comparación de dos medias y ANOVA de una vía para comparación de más de dos medidas). Las variables con distribución no Gaussiana fueron presentadas en forma de medianas y de los respectivos intervalos interquartílicos y las pruebas $U$ de Mann Whitney y Kruskal Wallis fueron empleadas para comparación de dos o más medianas, respectivamente.

La correlación lineal de Pearson o Spearman se utilizó para evaluar la relación entre el TAV y la razón TAV/TAS con los parámetros cardio-metabólicos. El análisis de regresión lineal simple se empleó para evaluar la capacidad predictiva de la razón TAV/TAS para explicar las alteraciones cardiometabólicas. Se estableció una significación estadística cuando el valor $\mathrm{p}<0,05$.

El protocolo de ese estudio se pautó en las normas éticas para investigación involucrando a seres humanos, constantes en la resolución 466/12 del Consejo Nacional de Salud, y fue sometido a la evaluación del Comité de Ética e Investigación en Seres Humanos de la Universidad de Pernambuco (UPE) aprobado con el número de protocolo 271.400/2013. Los individuos fueron previamente informados de los objetivos de la investigación, así como de los métodos adoptados $y$, mediante su consentimiento, firmaron un término de consentimiento libre y aclarado.

\section{RESULTADOS}

Se evaluaron 147 pacientes con promedio de edad $52,7 \pm 13,2$ años y predominio del sexo femenino (el $71,7 \%$ ). La prevalencia de hipertensión y diabetes fue del 59,7\% y $26,4 \%$, respectivamente. El estado nutricional según el IMC indicó el 2,2\% de pacientes con bajo peso y el $76,8 \%$ de pacientes con sobrepeso.

En la tabla 1, se observa una elevada media de la razón TAV/TAS para ambos sexos: $0,79 \pm 0,29$ para hombres y $0,54 \pm 0,22$ para mujeres, indicando una elevada predisposición para la acumulación de grasa visceral. Además, se identificó que para un mismo promedio de edad, incluso IMC y nivel de adiposidad abdominal subcutánea, los individuos del sexo masculino presentaron mayor concentración de grasa visceral $(p<0,001)$, mayor razón TAV/TAS $(p<0,001)$ y una mediana de puntuación de calcio $(p=0,033)$. Los parámetros metabólicos fueron similares en hombres y mujeres, excepto el HDL-c que fue superior en el sexo femenino $(p=0,010)$.

Se observó una asociación entre la edad y el TAV ( $r=$ $0,174 ; p=0,038)$ y la razón TAV/TAS $(r=0,331 ; p<0,001)$ y una correlación inversa con el TAS $(r=-0,303 ; p<0,001)$. El IMC presentó una fuerte correlación con la grasa subcutánea 
$(\mathrm{r}=0,848, \mathrm{p}<0,001)$ y correlación inversa con la razón TAV/TAS ( $r=-0,296 ; p<0,001)$. La CA presentó una fuerte relación con el TAS en comparación con el TAV (Tabla 2).

EI TAV aislado fue el componente que presentó mayor correlación con parámetros cardiometabólicos (siete parámetros), mientras que el TAS y la razón TAV/ TAS presentaron correlación con solo tres parámetros cardiometabólicos cada uno. El TAV presentó correlación directa con Colesterol no HDL, TG, razón TG/HDL, glicemia, $\mathrm{Hb}$ glicosilada y PCC $(\mathrm{p}<0,05)$ e inversa con el HDL-C $(p=0,001)$. El TAS presentó correlación directa con el TG $(p=0,011)$ y la proporción TG/HDL $(p=0,002)$ e inversa con el HDL-c $(p=0,002)$. La razón TAV/TAS, a su vez, fue correlacionada directamente con el perfil glicídico y con el PCC $(p<0,05)$ (Tabla 2).
En comparación con las alteraciones cardiometabólicas, según la concentración de TAV y la razón TAV/TAS, se identificó que las mujeres con mayor concentración de TAV (mayor tercil $\left(>283,6 \mathrm{~cm}^{2}\right)$ presentaron menor HDL-c $(p=0,021)$, mayor TG/HDL $(p<$ $0,001)$, mayores niveles de TG $(p<0,01)$, mayor glicemia $(p=0,009)$, mayor $\mathrm{Hb}$ glicosilada $(p=0,008)$ y mayor PCC $(p=0,034)$. No se verificó ninguna diferencia en los parámetros cardiometabólicos cuando estratificada la razón TAV/TAS en tercis (Tabla 3).

En el sexo masculino, los hombres en el mayor tercil de TAV $\left(>380,6 \mathrm{~cm}^{2}\right)$ presentaron mayores medianas de TG $(p=0,004)$ y razón TG/HDL $(p=0,007)$. En relación a la razón TAV/TAS, se observaron resultados equivalentes para los parámetros cardiometabólicos (Tabla 4).

\section{Tabla 1}

Comparación de los parámetros metabólicos, estado nutricional, puntuación de calcio coronario y tejido adiposo abdominal visceral (TAV) y subcutáneo (TAS) según sexo, en adultos atendidos ambulatoriamente en hospital en el Nordeste brasileño $(n=145)$.

\begin{tabular}{|c|c|c|c|}
\hline Variables & Hombres $(n=41)$ & Mujeres $(n=104)$ & Valor $p$ \\
\hline Edad* & $53,6 \pm 13,7$ & $52,3 \pm 13,0$ & 0,597 \\
\hline $\mathrm{HA}(\mathrm{n}, \%)^{\ddagger}$ & $26(63,4 \%)$ & $60(58,3 \%)$ & 0,569 \\
\hline $\mathrm{DM}(\mathrm{n}, \%)^{\ddagger}$ & $10(24,4 \%)$ & $28(27,2 \%)$ & 0,731 \\
\hline IMC (promedio/DS)* & $30,0 \pm 5,7$ & $31,5 \pm 6,2$ & 0,199 \\
\hline TAV (promedio /DS)* & $329,5 \pm 115,6$ & $248,9 \pm 77,8$ & $<0,001$ \\
\hline TAS (promedio/DS)* & $446,7 \pm 157,2$ & $496,4 \pm 155,7$ & 0,088 \\
\hline TAV/TAS (promedio/DS)* & $0,79 \pm 0,29$ & $0,54 \pm 0,22$ & $<0,001$ \\
\hline CA (promedio/DS)* & $105,7 \pm 15,2$ & $100,1 \pm 13,7$ & 0,039 \\
\hline CT (promedio/DS)* & $195,9 \pm 56,0$ & $208,1 \pm 48,8$ & 0,193 \\
\hline LDL -c (promedio/DS)* & $125,6 \pm 50,3$ & $128,6 \pm 41,8$ & 0,717 \\
\hline HDL-c (promedio/DS)* & $43,9 \pm 11,3$ & $50,0 \pm 13,2$ & 0,010 \\
\hline TG $(\text { mediana/ IQ) })^{+}$ & $118,1(83,2-183,1)$ & $134,2(92,8-183,7)$ & 0,282 \\
\hline TG/HDL-c (mediana/IQ) ${ }^{+}$ & $2,7(1,8-4,5)$ & $2,9(1,7-4,3)$ & 0,845 \\
\hline Col no-HDL (promedio/DS) & $151,9 \pm 54,7$ & $158,1 \pm 479$ & 0,528 \\
\hline 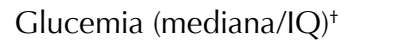 & $100,3(94,0-113,9)$ & $96,8(88,7-110,0)$ & 0,100 \\
\hline $\mathrm{Hb}$ glucosa (mediana /IQ) ${ }^{+}$ & $6,2(6,0-6,8)$ & $6,3(5,8-7,0)$ & 0,775 \\
\hline 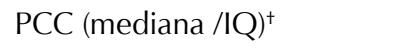 & $1,9(0-51,1)$ & $0,0(0-52)$ & 0,033 \\
\hline
\end{tabular}


Tabla 2

Correlación entre las variables estudiadas con tejido adiposo abdominal visceral (TAV), subcutáneo (TAS) y razón

TAV/TAS, en ambos sexos, en adultos atendidos ambulatoriamente en hospital en el Nordeste brasileño ( $\mathrm{n}=145)$

\begin{tabular}{|c|c|c|c|c|c|c|}
\hline \multirow[b]{2}{*}{ Variables } & \multicolumn{2}{|c|}{ TAV } & \multicolumn{2}{|c|}{ TAS } & \multicolumn{2}{|c|}{ TAV/TAS } \\
\hline & $\mathbf{r}$ & Valor $p$ & $\mathbf{r}$ & Valor $p$ & $\mathbf{r}$ & Valor $p$ \\
\hline Edad* & 0,174 & 0,038 & $-0,303$ & $<0,001$ & 0,331 & $<0,001$ \\
\hline IMC* & 0,325 & $<0,001$ & 0,848 & $<0,001$ & $-0,296$ & $<0,001$ \\
\hline $\mathrm{CA}^{*}$ & 0,540 & $<0,001$ & 0,759 & $<0,001$ & $-0,118$ & 0,168 \\
\hline $\mathrm{CT}^{*}$ & 0,113 & 0,179 & 0,012 & 0,884 & 0,090 & 0,285 \\
\hline HDL-c* & $-0,284$ & 0,001 & $-0,253$ & 0,002 & $-0,033$ & 0,693 \\
\hline LDL-c* & 0,101 & 0,232 & 0,052 & 0,539 & 0,061 & 0,474 \\
\hline $\begin{array}{l}\text { Col no- } \\
\mathrm{HDL}^{*}\end{array}$ & 0,190 & 0,024 & 0,078 & 0,354 & 0,101 & 0,231 \\
\hline $\mathrm{TG}^{+}$ & 0,379 & $<0,001^{\circ}$ & 0,214 & 0,011 & 0,078 & 0,355 \\
\hline $\mathrm{TG} / \mathrm{HDL}^{-\mathrm{C}^{+}}$ & 0,431 & $<0,001$ & 0,262 & 0,002 & 0,090 & 0,287 \\
\hline Glucemia $^{+}$ & 0,273 & 0,001 & 0,021 & 0,809 & 0,186 & 0,028 \\
\hline $\mathrm{Hb}$ glucosa ${ }^{+}$ & 0,237 & 0,006 & $-0,019$ & 0,827 & 0,172 & 0,047 \\
\hline $\mathrm{PCC}^{+}$ & 0,280 & 0,003 & 0,056 & 0,558 & 0,201 & 0,033 \\
\hline
\end{tabular}

Tabla 3

Análisis comparativo de edad y parámetros antropométricos según tercis del tejido adiposo visceral (TAV) y razón entre TAV y tejido adiposo subcutáneo (TAS) en mujeres atendidas ambulatoriamente en hospital en el Nordeste brasileño.

\begin{tabular}{|c|c|c|c|c|c|c|}
\hline \multirow[b]{2}{*}{ Variables } & \multirow[b]{2}{*}{$\begin{array}{l}1^{\circ} / 2^{\circ} \text { tercil } \\
\left(<283,6 \mathrm{~cm}^{2}\right)\end{array}$} & \multirow{2}{*}{$\begin{array}{c}\text { TAV } \\
>\text { tercil } \\
\left(>283,6 \mathrm{~cm}^{2}\right)\end{array}$} & \multirow[b]{2}{*}{ Valor $\mathbf{p}^{*}$} & \multicolumn{3}{|c|}{ TAV/TAS } \\
\hline & & & & $\begin{array}{c}1^{\circ} / 2^{\circ} \text { tercil } \\
(<0,62)\end{array}$ & $\begin{array}{l}>\text { tercil } \\
(>0,62)\end{array}$ & Valor $\mathbf{p}^{*}$ \\
\hline Edad* & $51,2 \pm 13,9$ & $54,2 \pm 10,9$ & 0,297 & $51,5 \pm 13$ & $54,2 \pm 13$ & 0,270 \\
\hline IMC* & $30,3 \pm 5,8$ & $34,3 \pm 6,0$ & 0,002 & $32,3 \pm 6,4$ & $30 \pm 5,4$ & 0,089 \\
\hline $\mathrm{CA}^{*}$ & $96,7 \pm 13,8$ & $107,2 \pm 10,6$ & $<0,001$ & $102,3 \pm 13,8$ & $96 \pm 12,5$ & 0,029 \\
\hline $\mathrm{CT}^{*}$ & $207,4 \pm 45,8$ & $211,6 \pm 54,8$ & 0,686 & $205 \pm 43,6$ & $215,2 \pm 58,1$ & 0,355 \\
\hline HDL-c* & $52,3 \pm 14$ & $45,8 \pm 10,4$ & 0,021 & $50,3 \pm 12,4$ & $50,1 \pm 15$ & 0,940 \\
\hline LDL-c* & $129 \pm 39,8$ & $128,6 \pm 46,9$ & 0,967 & $126,4 \pm 36,7$ & $133,8 \pm 51,6$ & 0,408 \\
\hline Col no-HDL* & $155,1 \pm 44,6$ & $165,8 \pm 54,4$ & 0,297 & $155,3 \pm 41,3$ & $165,2 \pm 60$ & 0,338 \\
\hline $\mathrm{TG} / \mathrm{HDL}-\mathrm{C}^{\dagger}$ & $2,5(1,7-3,2)$ & $4,4(3,5-4,9)$ & $<0,001$ & $2,8(1,9-3,4)$ & $3,5(1,6-4,4)$ & 0,377 \\
\hline $\mathrm{TG}^{+}$ & $114(94-149)$ & $182,9(150-212)$ & $<0,001$ & $130(140-156)$ & $146,6(100-201)$ & 0,401 \\
\hline Glucemia $^{+}$ & 94 (89-100) & 105 (96-115) & 0,009 & $96(91-100)$ & 103 (93-110) & 0,292 \\
\hline $\mathrm{Hb}$ glucosa ${ }^{+}$ & $6,2(5,9-6,4)$ & $6,8(6,2-7,2)$ & 0,008 & $6,3(5,9-6,5)$ & $6,4(6,1-7,0)$ & 0,181 \\
\hline $\mathrm{PCC}^{+}$ & $0(0-0)$ & $0(0-10,7)$ & 0,034 & $0(0-0)$ & $0(0-1,3)$ & 0,761 \\
\hline
\end{tabular}




\section{Tabla 4}

Análisis comparativo de edad y parámetros antropométricos según tercis del tejido adiposo visceral (TAV) y razón entre TAV y tejido adiposo subcutáneo (TAS) en hombres atendidos ambulatoriamente en hospital en el Nordeste brasileño.

\begin{tabular}{|c|c|c|c|c|c|c|}
\hline Variables & $\begin{array}{l}1^{\circ} / 2^{\circ} \text { tercil } \\
\left(<380,6 \mathrm{~cm}^{2}\right)\end{array}$ & $\begin{array}{c}\text { TAV } \\
>\text { tercil } \\
\left(>380,6 \mathrm{~cm}^{2}\right)\end{array}$ & Valor $\mathbf{p}^{*}$ & $\begin{array}{c}1^{\circ} / 2^{\circ} \text { tercil } \\
(<0,86)\end{array}$ & $\begin{array}{c}\text { TAV/TAS } \\
>\text { tercil } \\
(>0,86)\end{array}$ & Valor $\mathbf{p}^{*}$ \\
\hline Edad* & $52,1 \pm 15,1$ & $56,7 \pm 10,3$ & 0,326 & $48,9 \pm 13,5$ & $61,8 \pm 9,9$ & 0,003 \\
\hline IMC* & $189 \pm 60,8$ & $209 \pm 44,2$ & 0,283 & $188,6 \pm 62,8$ & $208,5 \pm 40,4$ & 0,224 \\
\hline $\mathrm{CA}^{*}$ & $46 \pm 12,1$ & $39,9 \pm 8,3$ & 0,103 & $44,7 \pm 12,9$ & $42,6 \pm 8,0$ & 0,589 \\
\hline $\mathrm{CT}^{*}$ & $120,4 \pm 50,3$ & $135,6 \pm 50,7$ & 0,367 & $121,7 \pm 58$ & $132,4 \pm 34$ & 0,462 \\
\hline HDL-C* & $140,0 \pm 57,8$ & $169,1 \pm 45,4$ & 0,151 & $143,9 \pm 60,2$ & $165,9 \pm 42$ & 0,179 \\
\hline LDL-C* & $27,7 \pm 4,4$ & $34,3 \pm 5,4$ & $<0,001$ & $30,2 \pm 6,3$ & $29,8 \pm 4,9$ & 0,824 \\
\hline Col no-HDL* & $99,2 \pm 12,6$ & $117,3 \pm 12,5$ & $<0,001$ & $105,5 \pm 16,8$ & $105,7 \pm 12,5$ & 0,977 \\
\hline $\mathrm{TG} / \mathrm{HDL}-\mathrm{C}^{\dagger}$ & 100 (94-114) & 105 (95-120) & 0,577 & $98(93-114)$ & $108(100-117)$ & 0,149 \\
\hline $\mathrm{TG}^{+}$ & $6,2(5,7-6,5)$ & $6,3(6,1-7,3)$ & 0,409 & $6,2(5,7-6,4)$ & $6,4(6,1-7,3)$ & 0,185 \\
\hline Glucemia $^{+}$ & 99 (74-129) & $174(114-312)$ & 0,004 & 101 (73-139) & $129(93-241)$ & 0,086 \\
\hline $\mathrm{Hb}$ glucosa ${ }^{+}$ & $2,3(1,7-3,2)$ & $4,3(2,3-9,5)$ & 0,007 & $2,6(1,7-4,1)$ & $3,2(2,1-5,0)$ & 0,221 \\
\hline $\mathrm{PCC}^{+}$ & $0(0-31,7)$ & $47,9(0-279)$ & 0,107 & $0(0-40)$ & $9,0(0-176)$ & 0,214 \\
\hline
\end{tabular}

\section{DISCUSIÓN}

La obesidad, principalmente la abdominal, predispone al individuo a una serie de factores de riesgo cardiovasculares por asociarse frecuentemente a condiciones como dislipidemia, hipertensión arterial, resistencia a la insulina y diabetes, que a su vez favorecen la ocurrencia de eventos cardiovasculares y otras complicaciones cardiometabólicas ${ }^{12}$. Los diferentes compartimentos de grasa predicen un riesgo cardiometabólico diferenciado, siendo el TAV el componente que presenta mayor efecto deletéreo sobre parámetros metabólicos y hemodinámicos ${ }^{4}$, presentando así mayor asociación con los tradicionales factores de riesgo cardiovasculares. EI TAV se considera el componente de tejido adiposo abdominal más metabólicamente activo, debido a la mayor respuesta a las catecolaminas y menor sensibilidad a la supresión de lipólisis mediada por insulina, además de liberar más ácidos grasos libres (AGL) directamente al hígado vía sistema porta ${ }^{5}$.

Observamos una elevada predisposición para la acumulación de grasa visceral en la población estudiada, con razón TAV/TAS superior a 0,4 en ambos sexos. El modelo de grasa ectópica establecido por la relación TAV/TAS sugiere que la energía excedente del balance energético entre el consumo de alimentos y el gasto calórico se almacena en compartimentos subcutáneos y cuando ese depósito entra en desequilibrio la energía excedente puede acumularse en compartimentos viscerales. Una sobrecarga de grasa en los adipocitos puede llevar a una escasez de grasa subcutánea, causando una acumulación de grasa visceral ${ }^{8}$.
De esa forma, algunos autores sugirieron que la razón TAV/TAS parece expresar mejor el riesgo del individuo de ser visceralmente obeso, porque refleja la proporción de acumulación de grasa en los sitios visceral y subcutáneo. La razón TAV/TAS observada en el presente estudio tanto para mujeres como hombres fue comparable al de otras investigaciones ${ }^{8,13}$, encontrándose los mayores valores en el sexo masculino.

La mayor concentración de TAV y la mayor razón TAV/TAS observada en el sexo masculino también fue observada por otros autores ${ }^{14,15}$ y pueden ser atribuidas a las evidentes diferencias en la composición corporal. Dichas diferencias pueden estar relacionadas a la partición regional de tejido adiposo, siendo los hombres más propensos a acumular tejido adiposo en la parte superior del cuerpo (tronco y abdomen), mientras que las mujeres suelen acumular grasa en la parte inferior del cuerpo (caderas y muslos), caracterizando los perfiles androide y ginoide, respectivamente ${ }^{16}$. Krotkiewski y cols. ${ }^{16}$, sugirieron que tales diferencias se derivan principalmente del número local de células de grasa, teniendo los hombres mayor concentración de células adiposas en la región abdominal, mientras que las mujeres presentan mayor concentración en la región glúteo-femoral, independientemente de la presencia o no de obesidad. Kuk y cols. ${ }^{17}$, demostraron que, para la misma circunferencia abdominal, los hombres presentaban mayor volumen de adiposidad visceral cuando comparados al sexo femenino. 
El marcado dimorfismo sexual en el patrón de distribución de grasa corporal en humanos está regulado por las diferencias hormonales ${ }^{18}$. Aunque no hay total comprensión sobre el papel que cada hormona desempeña en la modulación de la grasa corporal, algunas evidencias sugieren que los estrógenos tienen influencia significativa sobre la función del tejido adiposo y pueden ser un determinante importante en las diferencias de composición y estandarización de grasa corporal ${ }^{14}$. Esa hipótesis se ve reforzada por estudios que indican que la reducción de los niveles de estrógeno después de la menopausia se ha asociado con aumento de la adiposidad total, cambio en el patrón de distribución de grasa corporal y acumulación de grasa en el compartimento visceral ${ }^{19}$.

La correlación entre la edad y la concentración de TAV y la razón TAV/TAS observada en esta investigación es algo que está bien establecido en la literatura. En una muestra de voluntarios holandeses (hombres y mujeres), se verificó que la edad tiene un fuerte correlato para la acumulación selectiva de tejido adiposo abdominal, según lo estimado por el aumento de la circunferencia de la cintura ${ }^{20}$. Zamboni y cols. ${ }^{21}$, mostraron una correlación positiva entre la edad y la adiposidad visceral, así como una correlación negativa entre la edad y el TAS. Se sabe que la acumulación de grasa visceral aumenta con el avance de la edad como consecuencia de cambios relacionados con la distribución adiposa en los tejidos $^{14}$. La masa libre de grasa disminuye y la masa grasa generalmente aumenta, con almacenamiento preferencial en los sitios anatómicos intraabdominal e intramuscular, en lugar de la región subcutánea, como generalmente ocurre en el adulto joven ${ }^{22}$.

La correlación directa del IMC con el TAS e inversa con la razón TAV/TAS encontrada en el presente estudio refleja la limitación de ese parámetro para distinguir masa grasa de la masa magra y por no reflejar la distribución de grasa corporal ${ }^{23}$. Un aspecto importante a considerar en la evaluación del adulto es la distribución de la grasa corporal, independientemente del valor de IMC encontrado, siendo la obesidad abdominal el parámetro indicado como mejor predictor del riesgo coronario elevado ${ }^{23,24}$. El IMC es comúnmente utilizado para evaluar la adiposidad, pero es un parámetro que proporciona poca o ninguna información sobre la localización anatómica del exceso de grasa almacenada ${ }^{8}$. La distribución ectópica de grasa se considera mejor predictora de riesgo metabólico, independientemente de la cantidad absoluta de grasa ${ }^{8}$. Otros autores también relataron una débil o inexistente correlación entre la razón TAV/TAS e IMC ${ }^{8,25}$.

En nuestros hallazgos, la circunferencia abdominal (CA) presentó una correlación más fuerte con el TAS en comparación con el TAV y no presentó asociación con la razón TAV/TAS, indicando que ese parámetro puede ser útil para reflejar el adiposo abdominal total, pero no es potencialmente capaz de reflejar el depósito de grasa visceral y la propensión de almacenar grasa en ese compartimento, expresando predominantemente el contenido abdominal de grasa subcutánea. Ese hallazgo corrobora los datos presentados previamente.

En cuanto a los parámetros cardiometabólicos, nuestra investigación mostró resultados diferentes de los encontrados en la literatura actual, con el TAV aislado presentando correlación con un mayor número de parámetros evaluados cuando comparados a la razón TAV/TAS, que presentó correlación con solo tres parámetros cardiometabólicos. Al estudiar la correlación del TAV o TAS con el riesgo cardiometabólico, se ha concensuado que el TAV es un mejor predictor de alteraciones que el TAS. Sin embargo, todavía son escasos los estudios que hayan evaluado la asociación de la razón TAV/TAS como factores de riesgo cardiometabólico.

En una de las pocas investigaciones existentes, Kaess y cols. ${ }^{8}$, evaluando a 3.223 individuos participantes del Framingham Heart Study, mostraron que la razón TAV/TAS fue un predictor del riesgo cardiometabólico superior al IMC y TAV aislado, sugiriendo que la propensión a almacenar energía en el compartimento visceral o subcutáneo es un correlato de desregulación metabólica, independiente de la obesidad general y de la masa de grasa visceral. He y cols. ${ }^{26}$, en una muestra de 437 individuos, observaron que en las mujeres $(n=197)$ la razón TAV/TAS se asoció independientemente a la agrupación de factores de riesgo cardiometabólico, mientras que en los hombres el TAV aislado fue un marcador más potente de alteraciones en comparación con la razón TAV/TAS.

La razón TAV/TAS parece proporcionar información independiente de las medidas de patrón de adiposidad generalizada o abdominal. La cantidad absoluta del TAV parece proporcionar poca información sobre la distribución relativa del tejido abdominal ${ }^{8}$, no reflejando la predisposición al almacenamiento visceral o subcutáneo. Como los estudios disponibles no apuntan hacia una dirección única, es necesario que la razón TAV/TAS sea más profundamente estudiada para evaluar si es posible representar un mejor indicador de riesgo para rastrear a los individuos con perfil cardiometabólico alterado 7,8 .

En relación al perfil glucídico, en el presente estudio, la razón TAV/TAS presentó una asociación directa con glicemia de ayuno y hemoglobina glicosilada. Kaess y cols. ${ }^{8}$, encontraron mayor prevalencia de glicemia de ayuno alterada, resistencia a la insulina (definida por HOMA-IR) y diabetes en mujeres con mayor proporción de la razón TAV/ TAS. En los hombres, los resultados fueron similares, aunque no alcanzaron significancia estadística. Gastaldelli y cols. ${ }^{25}$, relataron que la razón TAV/TAS fue asociada a la glicemia de ayuno, mientras que el TAV y el TAS aisladamente no se correlacionaron. Miazaki y DeFronzo ${ }^{27}$ mostraron que, en una muestra de 36 hombres portadores de DM2, la razón TAV/TAS se correlacionó con la producción endógena de glucosa. Las citocinas inflamatorias secretadas, como IL-6 y TNF- $\alpha$, interfieren en la señalización intracelular de la insulina, comprometen la función endotelial y el metabolismo postprandial ${ }^{28}$. El hecho de que el TAV libera mayores 
concentraciones de citocinas inflamatorias en la circulación portal expone el hígado a la acumulación de grasa, estimula la liberación de apolipoproteína B (principal componentes de las lipoproteínas aterogénicas), reduce la sensibilidad a la insulina y aumenta la cantidad de glucosa plasmática ${ }^{29}$.

La evaluación del PCC nos indica la presencia calcificaciones coronarias, que se asocian a mayor riesgo para eventos coronarios. La correlación de la razón TAV/TAS y del TAV aislado con el PCC observada en nuestros hallazgos es un resultado que corrobora los resultados presentados por Bouchi y cols. ${ }^{30}$. En esa investigación, realizada con individuos japoneses con DM2, la razón TAV/TAS presentó correlaciones positivas y significativas con aumento del espesor de la capa íntima (ECI). Recientemente, se reportó que el TAV podría estar asociado a la formación de placa coronaria en pacientes diabéticos, considerándose un factor de riesgo relevante para el desarrollo de aterosclerosis carotídea. Los individuos con alta razón TAV/TAS presentan una menor capacidad para acumular exceso de energía en el depósito subcutáneo, llevando a una acumulación ectópica dentro del músculo, con consecuente aumento del riesgo de aterosclerosis carotídea ${ }^{30}$.

La cuestión racial es un aspecto relevante a ser considerado en el análisis de la composición corporal y diferentes resultados en poblaciones distintas pueden ser parcialmente explicados bajo ese aspecto. La población brasileña presenta características raciales específicas, marcada por un gran y complejo mestizaje. Por tanto, los resultados de estudios desarrollados en otros grupos étnicos no deben ser generalizados para todas las poblaciones. Eso refuerza la necesidad de que más estudios involucrando los componentes de tejido adiposo intraabdominales sean realizados en la población brasileña, donde todavía son muy escasos. Otra limitación del estudio se refiere al diseño transversal, imposibilitando cualquier inferencia causal definitiva. A pesar de ello, se debe considerar que son datos iniciales sobre el tema en la población brasileña, con la adopción de un método muy preciso, considerado patrón oro, para cuantificación de la grasa intraabdominal.

\section{CONCLUSIÓN}

La razón TAV/TAS presentó correlación con el perfil glucídico y PCC y puede considerarse un marcador de riesgo cardiometabólico importante. Sin embargo, la concentración de TAV aislada se correlacionó con siete parámetros cardiometabólicos, siendo en esa investigación un predictor más potente que la razón TAV/TAS para indicar alteraciones cardiometabólicas. La hipótesis planteada en estudios previos de que la razón TAV/TAS sería un predictor más potente que la concentración de TAV aislada para predecir alteraciones metabólicas no fue confirmada en esa investigación.

En vista de la importancia de la obesidad visceral en la génesis de varias comorbilidades, resulta claramente demostrada la importancia de acumular más evidencia sobre la evaluación de la predisposición de los individuos de acumular TAV, además de investigar el papel de la razón TAV/TAS como marcador de alteraciones cardiometabólicas en comparación con otras medidas.

Apoyo financiero: Autofinanciado.

Conflicto de interés: Ninguno.

\section{BIBLIOGRAFÍA}

1. World Health Organization. Obesity: preventing and managing the global epidemic. Ginebra, 2000.

2. Engl I, Sturm W, Sandhofer A, Kaser S, Tschoner A, Tatarczk $T$, et al. Effect of pronounced weight loss on visceral fat, liver steatosis and adiponectin isoforms. Eur J Clin Invest 2008; 38(4): 238-244.

3. Srdic B, Stokic E, Korac A, Ukropina M, Velickovic K, Breberina M. Morphological characteristics of abdominal adipose tissue in normalweight and obese women of different metabolic profiles. Exp Clin Endocrinol Diabetes 2010; 118(10): 713-718.

4. Vasques AC, Rosado L, Rosado G, Ribeiro RC, Franceschini S, Geloneze B. Anthropometric indicators of insulin resistance. Arq Bras Cardiol 2010; 95(1): 14-23.

5. Hermsdorff HHM, Monteiro JBR. Visceral, subcutaneous or intramuscular fat: where is the problem? Arq Bras Endocrinol Metabol 2004; 48(6): 803-811.

6. Rajala MW, Scherer PE. Minireview: The adipocyte- at the crossroads of energy homeostasis, inflammation, and atherosclerosis. Endocrinol 2003; 144(9): 3765-3773.

7. Ribeiro-Filho FF, Mariosa LS, Ferreira SRG, Zanella MT. Visceral fat and metabolic síndrome: More tan a simple association. Arq Bras Endocrinol Metab 2006; 50(2): 230-238.

8. Kaess BM, Pedley A, Massaro JM, Murabito J, Hoffmann U, Fox CS. The ratio of visceral to subcutaneous fat, a metric of body fat distribution, is a unique correlate of cardiometabolic risk. Diabetol 2012; 55(10): 2622-2630.

9. Borkan GA, Gerzof SG, Robbins AH, Hults DE, Silbert CK, Silbert JE. Assessment of abdominal fat content by computed tomography. Am J Clin Nut 1982; 36: 172-177.

10. World Health Organization. Obesity: Preventing and managing the global epidemic. Report of a WHO Consultation on Obesity. Geneva; 1998.

11. Lee SY, Chang HJ, Sung J, Kim KJ, Shin S, Cho IJ, et al. The impact of obesity on subclinical coronary atherosclerosis according to the risk of cardiovascular disease. Obesity (Silver Spring) 2014; 22(7): 1762-1768.

12. Vasques ACJ, Priore SE, Rosado LEFPL, Franceschini SCC. The use of anthropometric measures to asseess visceral fat accumulation. Rev Nutr 2010; 23: 107-118.

13. Yeoh AJ, Pedley A, Rosenquist KJ, Hoffmann U, Fox CS. The Association between subcutaneous fat density and the propensity to store fat viscerally. I Clin Endocrinol Metab 2015; 100(8): 1056-1064.

14. Tchernof A, Després JP. Pathophisiology of human visceral obesity: an update. Physiol Rev 2013; 93: 359-404.

15. Porter SA, Massaro JM, Hoffmann U, Vasan RS, O"Donnel CJ, Fox CS. Abdominal subcutaneous adipose tissue: a protective fat depot? Diabetes Care 2009; 32: 1068-1075.

16. Krotkiewski M, Björntorp P, Sjöström L, Smith U. Impact of obesity on metabolism in men and women. Importance of regional adipose tissue distribution. J Clin Invest 1983; 72: 1150-1162.

17. Kuk LL, Lee S, Heymsfield SB, Ross R. Waist circumference and abdominal adipose tissue distribution: Influence of age 
and sex. Am / Clin Nutr 2005; 81: 1330-1334.

18. TChernof A, Després JP. Sex steroid hormones, sex hormonebinding globulin, and obesity in men and women. Horm Metab Res 2000; 32: 526-536.

19. Lovejoy JC, Champagne CM, de Jonge L, Xie H, Smith SR. Increased visceral fat and decreased energy expenditure during the menopausal transition. Int J Obes 2008; 32: 949-958.

20. Han TS, McNeill G, Seidell JC, Lean ME. Predicting intraabdominal fatness fromanthropometric measures: The influence of stature. Int J Obes Relat Metab Disord 1997; 21: 587-593.

21. Zamboni M, Armellini F, Milani MP, De Marchi M, Todesco $T$, Robbi R, Bergamo-Andreis IA, Bosello O. Body fat distribution in pre- and post-menopausal women: Metabolic and anthropometric variables and their inter-relationships. Int J Obes RelatMetab Disord 1992; 16: 495-504.

22. Cervi A, Franceschini SMC, Priore SE. Critical analysis of the use of the body mass index for the elderly. Rev Nutr 2005; 18(6): 765-775.

23. Brazilian Association for the Study of Obesity and Metabolic Syndrome 2009/2010 (ABESO). Brazilian Guidelines for Obesity, 3rd edition. Brazil, 2009.
24. Wanderley EN, Ferreira VA. Obesity: a plural perspective. Ciênc e Saud Coletiv 2010; 15(1): 185-194.

25. Gastaldelli A, Sironi AM, Ciociaro D. Visceral fat and beta cell function in non-diabetic humans. Diabetol 2005; 48: 2090-2096.

26. He H, Ni Y, Chen J. Sex difference in cardiometabolic risk profile and adiponectin expression in subjects with visceral fat obesity. Transl Res 2011; 155: 71-77.

27. Miyazaki Y, DeFronzo RA. Visceral fat dominant distribution in male type 2 diabetic patients is closely related to hepatic insulin resistance, irrespective of body type. Cardiovasc Diabetol 2009; 8: 44.

28. Monteiro CA. Is obesity replacing or adding to undernutrition? Evidence from different social classes in Brazil. Public Health Nutr 2002; 5(1): 105-112.

29. Lear SA, Humphries KH, Kohli S, Frohlich JJ, Birmingham GB, Mancini GBJ. Visceral adipose tissue, a potential risk factor for carotid atherosclerosis. Stroke 2007; 38: 2422-2429.

30. Bouchi R, Takeuchi T, Akihisa M, Ohara N, Nakano Y, Nishitani $R$ et al. Hight visceral fat with low subcutaneous fat accumulation as a determinant of atherosclerosis in patients with type 2 diabetes. Cardiovasc Diabetol 2015; 14: 136. 